type I interferon, which prevented fatal viral infection of the nervous system.

The findings suggest that innate immunity - the immune system's first line of defence, which includes macrophages - has an unappreciated role in combating viral infections. Immunity http://dx.doi.org/ 10.1016/j.immuni.2012.01.013 (2012)

\section{MATERIALS SCIENCE}

\section{Competition looms for graphene}

Graphene - atomically thick sheets of carbon atoms arranged in a honeycomb pattern - has received much attention for its exceptional electrical properties, thought to arise from its hexagonal symmetry. But Daniel Malko and his colleagues at the University of ErlangenNuremberg in Germany propose that a similar material, graphyne, might be just as special - or even more so.

Graphynes are similar to graphene, the main difference being that they have triple bonds between some of their carbon atoms. Only small snippets of graphyne have been synthesized so far, but the authors performed a simulation showing that, like graphene, various forms of graphyne should have high electrical conductivity. The researchers note that not all graphynes have graphene's hexagonal honeycomb configuration. This suggests that many more two-dimensional materials with various symmetries and non-carbon structures - could have graphene-like properties. Phys. Rev. Lett. 108, 086804 (2012)

\section{CELL BIOLOGY}

\section{Sperm steer with calcium}

How winding a sperm's path is depends on the degree to which the concentration of calcium ions in the sperm changes.

Eggs attract sperm by sending out chemical cues that activate calcium-ion release in the sperm's tail, or flagellum. When Luis Alvarez and Benjamin Kaupp at the Center of Advanced European Studies and Research in Bonn, Germany, and their co-workers stimulated calcium surges in the flagella of seaurchin sperm, the sperm switched from swimming in circles to bouts of near-straight runs interlaced with turns. The curvature of the sperms' movement correlated with how quickly the concentration of calcium changed, regardless of the total concentration.

The researchers propose that two different calciumion-binding reactions could allow sperm to sense the rate of change in calcium levels. J. Cell Biol. http://dx.doi.org/ 10.1083/jcb.201106096 (2012)

\section{NEUROSCIENCE}

\section{Behind marijuana memory lapse}

Marijuana hampers shortterm memory by activating a signalling pathway between neurons and non-neuronal cells called astrocytes, work in mice suggests. This is surprising because astrocytes have mostly been considered to be just support cells.

Giovanni Marsicano at the French National Institute of Health and Medical Research in Bordeaux, Xia Zhang of the University of Ottawa in Canada and their colleagues found that marijuana's psychoactive ingredient, tetrahydrocannabinol (THC), weakens the connections, or synapses, between neurons in the hippocampus - a brain region crucial for memory formation. They tested the effects of THC on mice engineered so that either their neurons or their astrocytes lacked the $\mathrm{CB}_{1}$ receptor that $\mathrm{THC}$ acts on. In mice missing the astrocyte receptors, THC had no effect on hippocampal synapses or working memory, as shown in a behavioural test.

The authors propose that the activation of astrocyte

COMMUNITY CHOICE

The most viewed papers in science

\title{
Unintended antimicrobial effects
}

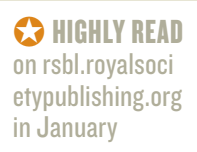

in January
As antibiotic resistance in microorganisms has risen, some scientists have suggested that short, synthetic amino-acid chains, or peptides, that kill invading pathogens could make a new therapeutic weapon. But Michelle Habets and Michael Brockhurst at the University of Liverpool, UK, highlight serious potential risks of this strategy.

The researchers exposed the pathogen Staphylococcus aureus - which causes a range of dangerous infections and has developed resistance to many antibiotics - to increasing levels of a synthetic peptide called pexiganan, a candidate treatment for diabetic leg-ulcer infection. Over several tens of generations, the bacteria evolved resistance to the peptide. Furthermore, some bacteria also evolved resistance to HNP-1, a natural human peptide that is an important part of innate immunity, which represents the immune system's first line of defence.

The authors say that such cross-resistance could undermine the innate immune system's ability to prevent superficial infections from progressing to life-threatening disease. Biol. Lett. http://dx.doi.org/10.1098/rsbl.2011.1203 (2012)
$\mathrm{CB}_{1}$ receptors by $\mathrm{THC}$ causes receptors for a compound called AMPA to be removed from the membranes of neurons, which is known to weaken synapses. Cell 148, 1039-1050(2012)

\section{VIROLOGY}

\section{Bats can carry} flu too

Fruit bats in

Guatemala

harbour a

strain of

influenza

virus,

researchers report expanding the known mammalian reservoir for the largely bird-borne influenza A.

Suxiang Tong of the US Centers for Disease Control and Prevention in Atlanta, Georgia, and her colleagues screened 316 bats from 21 species for a variety of viruses. Viral genome sequencing revealed that three little yellowshouldered bats (Sturnira lilium; pictured), were positive for influenza A. Analysis of two key proteins that are shared by all influenza strains, as well as other viral genes, showed that this strain - designated H17 - diverged from other known influenza viruses long ago.

The authors say that $\mathrm{H} 17$ seems to pose no immediate threat to humans. However,

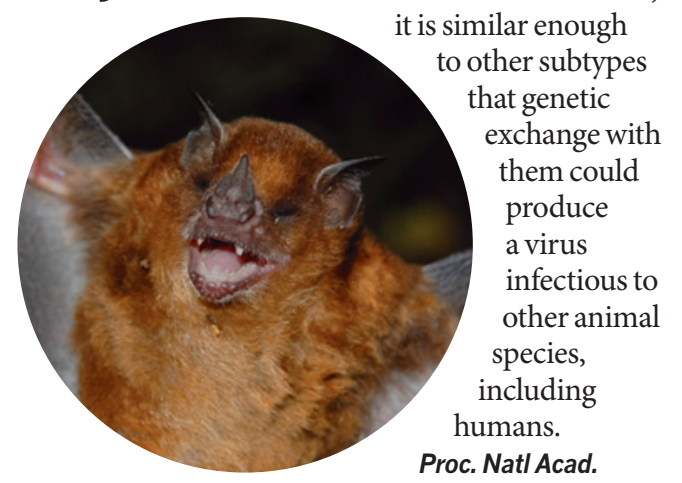

Sci. USA http://dx.doi. org/10.1073/pnas.1116200109 (2012)

For a longer story on this research, see go.nature.com/ fnjy2l

\section{$\rightarrow$ NATURE.COM}

For the latest research published by Naturevisit:

www.nature.com/latestresearch 